\title{
Development of an Aeroservoelastic Model for Gust Load Alleviation of the NASA Common Research Model Wind Tunnel Experiment
}

\author{
Nick Cramer* and Nhan Nguyen ${ }^{\dagger}$ \\ NASA Ames Research Center, Moffett Field, CA 94035
}

\begin{abstract}
As aircraft move to using composite materials as their primary structure they become lighter and more flexible as well. This presents some significant challenges in association with gust load alleviation. In this paper we develop an aeroservoelastic model for use in developing controllers that utilize distributed control surfaces for active gust load alleviation in a set of wind tunnel experiments. The model is based on an preexisting aeroelastic wing tunnel model and compares the baseline functionality to it. We also provide simple full state feedback simulations for the model.
\end{abstract}

\section{Introduction}

$\mathrm{T}$ HE design of aircraft are required to be able to with stand the loads applied to them from external gust for the sake of survivability.[1] As aircraft have continued to reduce their structural weight they are reducing the safety factor associated with gust so the investigation into active gust load alleviation has been steadily gaining importance over the past few decades. By applying gust load alleviation their are opportunities not only to increase the aircraft's safety factor but also reduce aircraft weight.[2]

There are quite a few different approaches for active gust load alleviation. One of the more common approaches is to use model predictive control and using proposed look ahead LIDAR methods to observe the future gust states and react to it appropriately. [3, 4] The SensorCraft used an Generalized Predictive Controller approach for their experiments with an AutoRegressive eXoegnenous input and impulse response model system identification rather successfully.

This experiment and the associated model that we develop in this paper are looking to take advantage of the variable camber continuous trailing edge technology as a means of multi-objective control included gust load alleviation. The double hinge configuration allows the model to change it's camber and has been shown to be an effective means of reducing drag.[5] There have been additional promising simulation results suggesting that the the VCCTEF design can effectively maintain good tracking while reducing modal response and gusts.[6] It has also shown promise with time-varying weight for multi-objective control of drag and maneuver load alleviation. These results are promising enough that they warrant experimental investigation.

This paper will develop and aeroservoelastic model for an wind tunnel experiment of multi-objective control. We will start by reviewing the general geometry that will be used for the testing then investigate the approach taken to build up this preliminary model comparing it to pre-existing tunnel models. Finally we will have some simulation results.

\section{Wing Geometry}

The wing geometry used for this experiment is NASA's Common Research Model which was developed to provide an open platform for research. The NASA Common Research Model (CRM) is a wide-body commercial aircraft with supercritical transonic wing. [7] Figure 1 shows an CAD rendering of the full sized CRM.

\footnotetext{
*Insert Job Title, Department Name, Address/Mail Stop, and AIAA Member Grade (if any) for first author

†NASA Ames Research Center, Research Scientist, AIAA Associate Fellow, nhan.t.nguyen@nasa.gov
} 


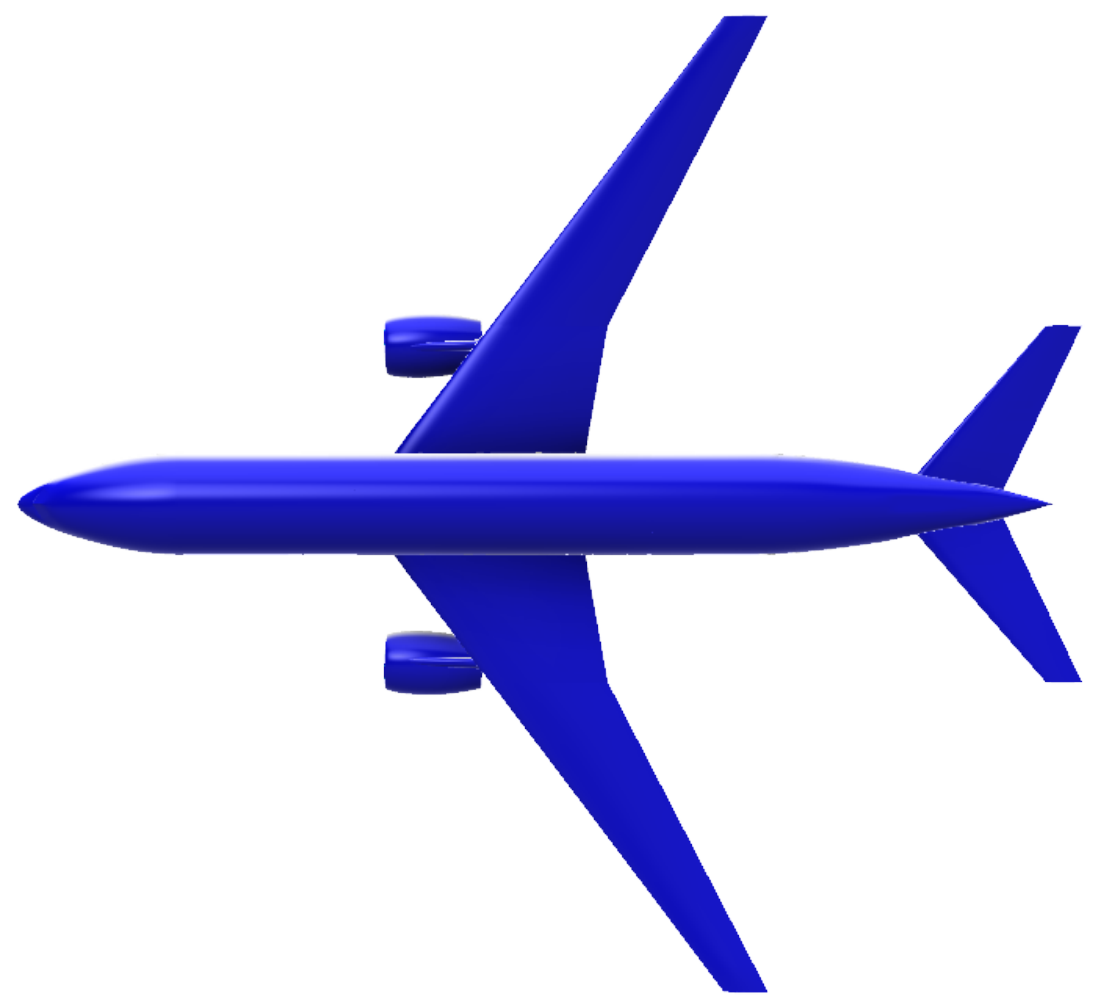

Fig. 1 CAD model of full scale CRM

This set of experiments and the model we are developing for them uses the an twist optimized version of the CRM wing with VCCTEF applied to it as the layout schematic in Figure 2 shows. The wing used was 7 foot half span and had 12 controller surfaces or 6 VCCTEFs. Further details of the actual tunnel model can be found in [8]. 


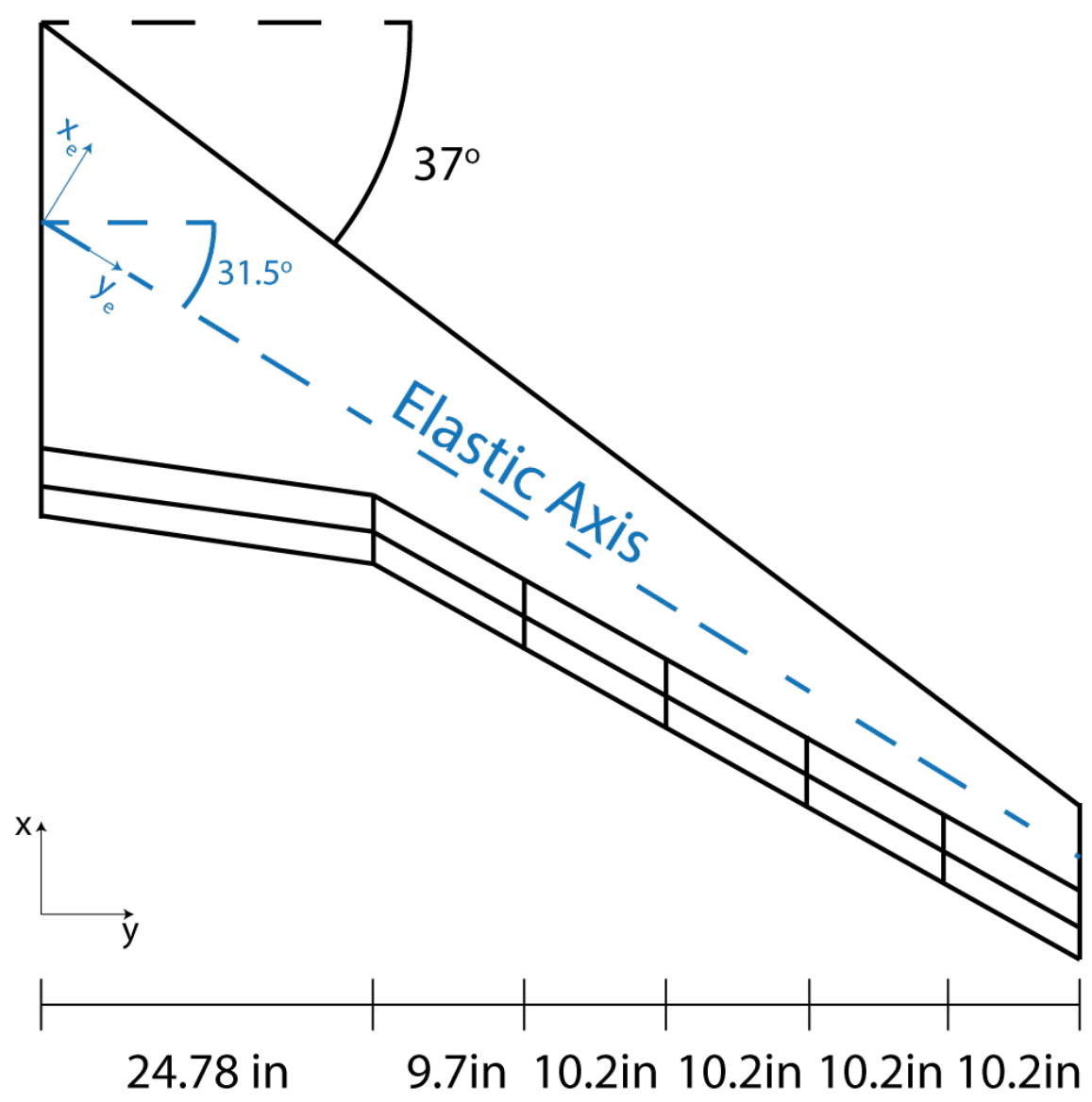

Fig. 2 Wind tunnel wing and flap layout

\section{Structural Modeling}

The structural model of the wing can be represented as a beam with flapwise or wing out-of-plane bending, w, chordwise or in-plane bending, $\mathrm{v}$, axial displacement, $\mathrm{u}$, and torsion $\theta$. The force and moment equilibrium equations for the representative beam are

$$
\begin{gathered}
\frac{\partial E A u_{y_{e}}}{\partial y_{e}}+f_{y_{e}}=0 \\
\frac{\partial G J \theta_{e}}{\partial y_{e}}+f_{y_{e}}=0 \\
\frac{\partial^{2}}{\partial y_{e}^{2}}\left(E I_{x_{e} x_{e}} w\right)=f_{z}-\frac{\partial m_{x_{e}}}{\partial y_{e}} \\
\frac{\partial^{2}}{\partial y_{e}^{2}}\left(E I_{z z} v\right)=f_{y_{e}}-\frac{\partial m_{z}}{\partial y_{e}}
\end{gathered}
$$

where $E$ is the modulus of elasticity, $I_{i i}$ is the area moment of inertia for the $i i$ axis, $y_{e}$ is along the elastic axis and $x_{e}$ is perpendicular with positive towards the leading edge, $f_{i}$ is the force in the $i$ direction, $m_{i}$ is the moment about the $i$ axis. 


\section{A. Finite Element Modeling}

The wing beam can be represented as an series of finite elements for the purpose of modeling. Using the same finite element formulation as used in previous works [9, 10] the beam can be broken into $N$ straight beam elements along the elastic axis. We can assume that the finite elements are following specified shape functions $N_{w}\left(y_{e}\right), N_{v}\left(y_{e}\right)$, and $N_{\theta}\left(y_{e}\right)$ where the $N_{w}$ and $N_{v}$ are Hermite shape functions and $N_{\theta}$ is a linear shape function with respect to the elastic axis $y_{e}$.

The system of finite elements for element $j$ can be represented as an elemental stiffness matrix

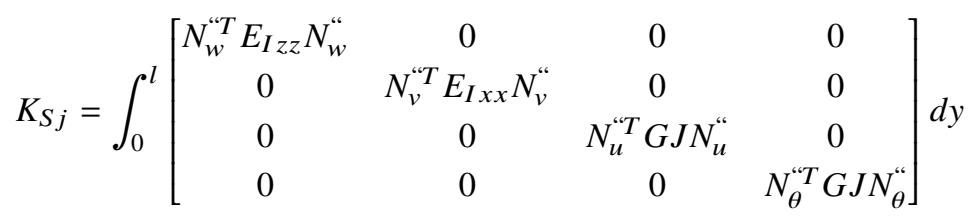

where $K_{S j}$ is the element stiffness matrix and the associated mass matrix is

$$
M_{S j}=\int_{0}^{l}\left[\begin{array}{cccc}
N_{w}^{T} \rho A N_{w} & 0 & 0 & -N_{w}^{T} \rho A e_{c g} N_{\theta} \\
0 & N_{v}^{T} \rho A N_{v} & 0 & 0 \\
0 & 0 & N_{u}^{T} \rho N_{u} & 0 \\
-N_{\theta}^{T} \rho A e_{c g} N_{w} & 0 & 0 & N_{\theta}^{T} \rho I_{x_{e} x_{e}} N_{\theta}
\end{array}\right] d y
$$

The local coordinate vectors are rearranged to be

$$
\mu_{j}=\left[\begin{array}{llllllllllll}
w_{1} & w_{y_{e_{1}}} & v_{1} & v_{y_{e_{1}}} & u_{y_{e_{1}}} & \theta_{e_{1}} & w_{2} & w_{y_{e_{2}}} & v_{2} & v_{y_{e_{2}}} & u_{y_{e_{2}}} & \theta_{e_{2}}
\end{array}\right]_{j}^{T}
$$

\section{B. Translation of Local Coordinates to Global Coordinates}

The section stiffness and mass matrices in Equations 5 and 6 are done in the local beam coordinates on the elastic axis. They need to be translated back into the global coordinate frame. This is done on an node by node basis where they are translated to global states by

$$
\mu_{j}=T_{j} p_{j}
$$

where,

$$
\overbrace{\left[\begin{array}{c}
w \\
w_{y_{e}} \\
v \\
v_{y_{e}} \\
u_{y_{e}} \\
\theta_{y_{e}}
\end{array}\right]_{j}}^{\mu_{j}}=\overbrace{\left[\begin{array}{cccccc}
\cos \Gamma_{j} & 0 & -\sin \Lambda_{j} \sin \Gamma_{j} & 0 & -\cos \Lambda_{j} \sin \Gamma_{j} & 0 \\
0 & \cos \Lambda_{j} & 0 & 0 & 0 & \sin \Lambda_{j} \\
0 & 0 & \cos \Lambda_{j} & 0 & -\sin \Lambda_{j} & 0 \\
0 & \sin \Lambda_{j} \sin \Gamma_{j} & 0 & \cos \Gamma_{j} & 0 & -\cos \Gamma_{j} \sin \Gamma_{j} \\
\sin \Gamma_{j} & 0 & \sin \Lambda_{j} \cos \Gamma_{j} & 0 & \cos \Lambda_{j} \cos \Gamma_{j} & 0 \\
0 & -\sin \Lambda_{j} \cos \Gamma & 0 & \sin \Gamma_{j} & 0 & \cos \Lambda_{j} \cos \Gamma_{j}
\end{array}\right]\left[\begin{array}{c}
\Delta z \\
\Delta \phi_{z} \\
\Delta x \\
\Delta \phi_{x} \\
\Delta y \\
\Delta \phi_{y}
\end{array}\right]_{j}}^{T_{j}}
$$

The $p_{j}$ 's can then be combined to get the total displacement vector

$$
p=\left[\begin{array}{lllllllllllll}
\Delta z_{1} & \Delta \phi_{z_{1}} & \Delta x_{1} & \Delta \phi_{x_{1}} & \Delta y_{1} & \Delta \phi_{y_{1}} & \ldots & \Delta z_{N+1} & \Delta \phi_{z_{N+1}} & \Delta x_{N+1} & \Delta \phi_{x_{N+1}} & \Delta y_{N+1} & \Delta \phi_{y_{N+1}}
\end{array}\right]
$$

The global structural mass and stiffness matrices can be assembled to correspond with the global displacement vector $p$ and are formed by

$$
M_{S}=\sum_{j=1}^{N} T_{j}^{T} M_{S_{j}} T_{j}
$$

and

$$
K_{S}=\sum_{j=1}^{N} T_{j}^{T} K_{S_{j}} T_{j}
$$


which results in a structural FEM system of equations

$$
M_{S} \ddot{p}+K_{S} p=F
$$

For the wind tunnel model a fixed root boundary condition can be applied by zeroing out all of the displacement at the root node and removing the associated cells for $M_{S}$ and $K_{S}$. From here on $M_{S}$ and $K_{S}$ will be referring to the structural mass and stiffness matrices with the fixed root boundary conditions applied.

\section{Structural Damping}

A structural damping factor is necessary to represent the natural energy dissipation of the wings constitute materials, internal friction, and various other damping factors. The viscous damping is represented by $\zeta_{s}$ where $\zeta_{s}=\zeta I$. In general each mode will have a unique damping factor but for initial simulations we will assume they all share the same value.

The structural damping matrix can be found by

$$
C_{s}=2 M_{S} X_{S} \zeta_{S} \Omega_{S} X_{S}^{-1}
$$

where $X_{S}$ is the eigenvector matrix of the matrix $M_{S}^{-1} K_{S}$ and $\Omega_{S}$ is the diagonal matrix of natural frequencies associated with the structural matrices.

\section{Modal Transformation and Reduction}

Combining equation 13 with 14 results in

$$
M_{s} \ddot{p}+C_{S} * \dot{p}+K_{S} p=F
$$

By using the eigenvector matrix $X_{S}$ presented earlier an modal transformation can be applied to Equation 15 Modal reduction can be performed during this process by truncating $X_{S}$ such that only the desired modes are remaining creating a reduced order modal matrix $\Phi$ resulting in the modal equation

$$
\Phi^{T} M_{S} \Phi \ddot{q}+\Phi^{T} C_{S} \dot{q}+\Phi^{T} K_{S} \Phi q=\Phi^{T} F
$$

From this point forward we will be using the general modal coordinates where $q$ are the general coordinates and

$$
\begin{gathered}
M_{m S}=\Phi^{T} M_{S} \Phi \\
K_{m S}=\Phi^{T} K_{S} \Phi \\
C_{m S}=\Phi^{T} C_{S} \Phi
\end{gathered}
$$

where $M_{m s}, K_{m S}, C_{m S}$ are the modal mass, stiffness, and damping matrices respectively.

\section{E. Structural Modes}

The model here was based on an existing wind tunnel model shown in [8] and we can compare the associated modes. Table 1 compares the modes of the GVT and NASTRAN results to the

We can see from Table 1 that the torsional modes were especially difficult to match. This was not associated with just the structure but also ensuring convergence of the aeroelastic model for the trim condition of the ASE. 


\begin{tabular}{|c|c|c|c|c|}
\hline Mode & Experiment & NASTRAN & MATLAB FEM & Description \\
\hline 1 & 2.8 & 2.8 & 2.96 & $1^{\text {st }}$ Bending \\
\hline 2 & 11.3 & 12.2 & 13.78 & $2^{\text {nd }}$ Bending \\
\hline 3 & 13.2 & 13 & 15.0 & In-Plane Bending + (Vertical) Bending \\
\hline 4 & 27 & 31 & 31.6 & Bending + Torsion \\
\hline 5 & 33 & 33 & 44.5 & $1^{\text {st }}$ Torsion \\
\hline 6 & 47 & 48 & 58.8 & In-Plane Bending \\
\hline 7 & 52 & 58 & 68.8 & $3^{\text {rd }}$ Bending \\
\hline 8 & 70 & 67 & 89.9 & $2^{\text {nd }}$ Torsion \\
\hline
\end{tabular}

Table 1 Comparison of MATLAB modes, NASTRAN, and experimental.

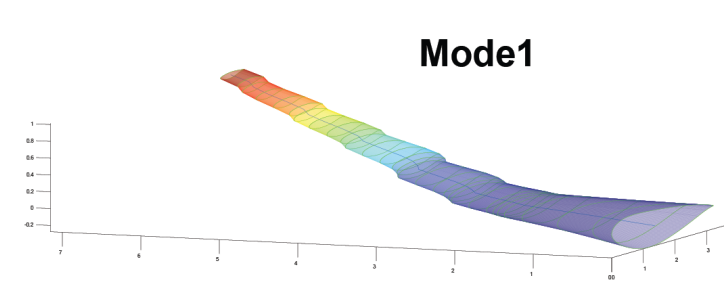

Mode3

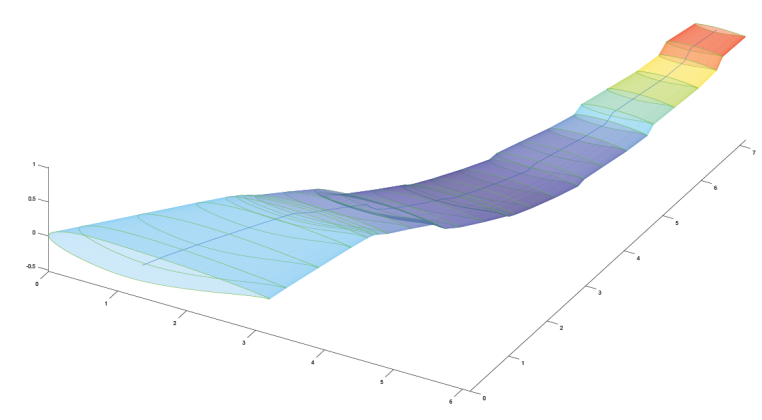

Mode5

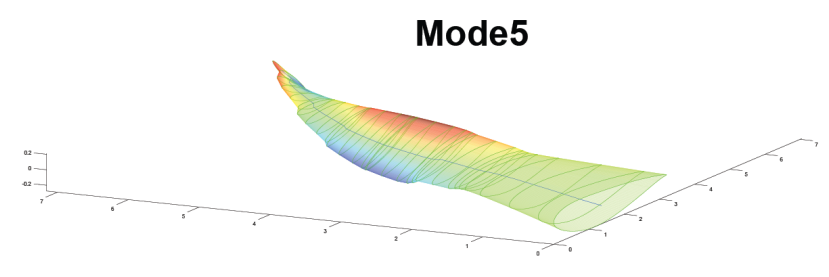

\section{Mode7}

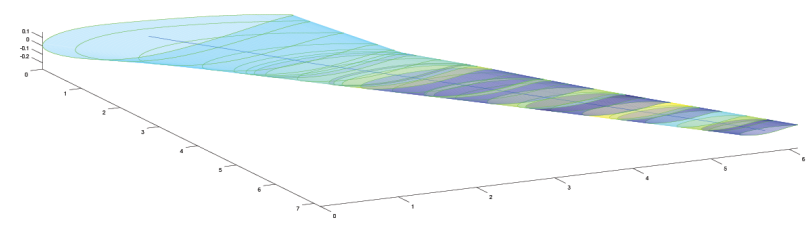

Mode2

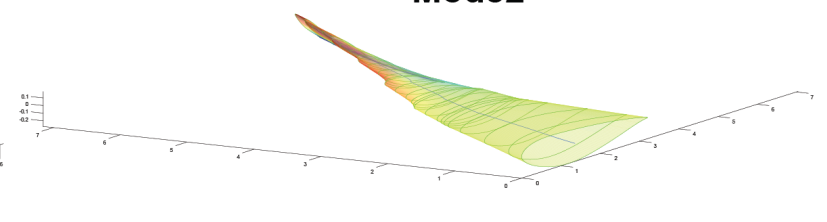

Mode4

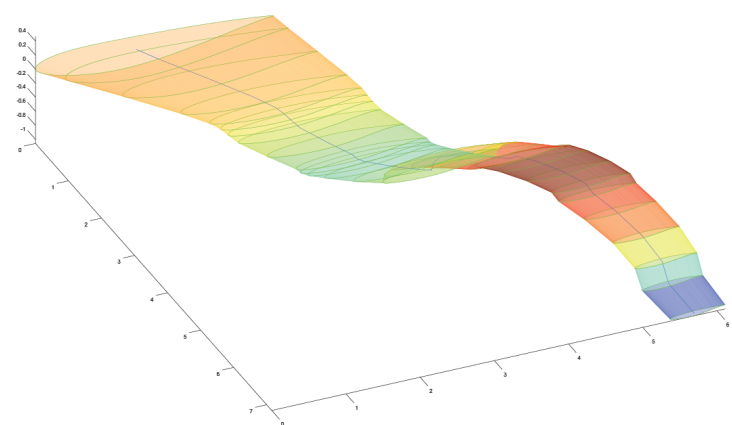

Mode6

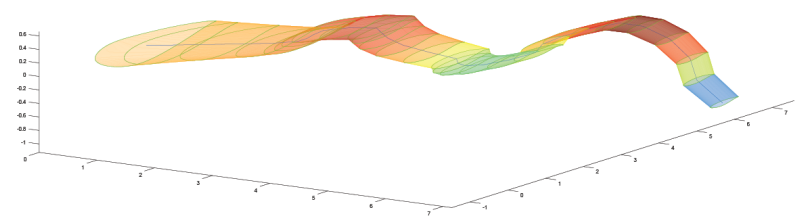

Mode8

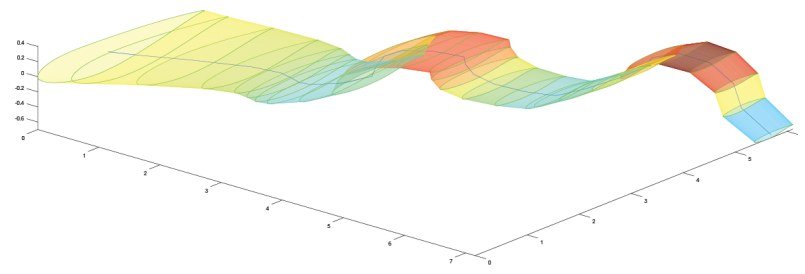

Fig. 3 Mode shapes of the wing tunnel CRM 
Figure 3 shows the equivalent MATLAB Beam model modes. They compare favorably to the NASTRAN mode shapes from [8].

\section{Aeroelasticity}

The aeroelastic modeling section will focus on the adaptation of the models from [9] and [10] to fit the wind tunnel model. In the final version of the paper a more comprehensive derivation of the aeroelastic components will be provided for completeness.

\section{A. Side Wall Balance Angle of Attack}

While the wind tunnel model does not have rigid body dynamics there are dynamics and aeroeleastic forces that are derived from the side wall balance angle of attack. The sidewall balance angle of attack can be computed as[11]

$$
\alpha_{s}(y)=-\frac{-z_{t}}{u \cos \Lambda}\left(1+\frac{x_{t}+u}{u \cos \Lambda}\right)
$$

where $z_{t}$ is the vertical velocity, $x_{t}$ is the additional stream-wise velocity and $u$ is airspeed. Equation 20 can be represented as an partial derivative

$$
\alpha_{s}(y)=\frac{\partial \alpha_{c}}{\partial 1}+\frac{\partial \alpha_{c}}{\partial a l p h a} \alpha+\frac{\partial \alpha_{c}}{\partial q_{r}} q_{r}
$$

where

$$
\begin{gathered}
\frac{\partial \alpha_{c}}{\partial 1}=-\tan \Lambda \sin \Gamma \\
\frac{\partial \alpha_{c}}{\partial a l p h a}=\frac{\cos \Gamma}{\cos \Lambda} \\
\frac{\partial \alpha_{c}}{\partial q_{r}}=\frac{y \cos \Gamma}{V_{\infty} \cos \Lambda}
\end{gathered}
$$

where $\alpha_{c}$ is the complete local angle of attack and $q_{r}$ is the rigid body pitch rate. The force vectors for the elemental components can then be generated using the same methodology using the Hermite $N_{w}$ and linear interpolations $N_{u}$ and $N_{\theta}$ which generates elemental matrices

$$
\begin{aligned}
& H_{\alpha_{j}}=\int_{0}^{l}\left[\begin{array}{c}
N_{w}^{T} \frac{\partial L}{\partial y \partial \alpha} \frac{\partial \alpha_{c}}{\partial \alpha}-\left.N_{w}^{c} \frac{e_{c}}{c} \frac{\partial m}{\partial y \partial \alpha} \frac{\partial \alpha_{c}}{\partial \alpha}\right|_{e_{c}} \tan \Lambda \\
\left.N_{v}^{T} \frac{\partial D}{\partial y \partial \alpha} \frac{\partial \alpha_{c}}{\partial \alpha}\right|_{e_{c}} \\
\left.N_{u}^{T} \frac{\partial D}{\partial y \partial \alpha} \frac{\partial \alpha_{c}}{\partial \alpha}\right|_{e_{c}} ^{\tan \Lambda} \\
-\left.N_{\theta}^{T} \frac{\partial m}{\partial y \partial \alpha} \frac{\partial \alpha_{c}}{\partial \alpha}\right|_{e_{c}}
\end{array}\right] d y \\
& H_{q_{r_{j}}}=\int_{0}^{l}\left[\begin{array}{c}
N_{w}^{T} \frac{\partial L}{\partial y \partial \alpha} \frac{\partial \alpha_{c}}{\partial q_{r}}-\left.N_{w}^{T} \frac{e_{c}}{c} \frac{\partial m}{\partial y \partial \alpha} \frac{\partial \alpha_{c}}{\partial q_{r}}\right|_{e_{c}} \tan \Lambda \\
\left.N_{v}^{T} \frac{\partial D}{\partial y \partial \alpha} \frac{\partial \alpha_{c}}{\partial q_{r}}\right|_{e_{c}} \\
\left.N_{u}^{T} \frac{\partial D}{\partial y \partial \alpha} \frac{\partial \alpha_{c}}{\partial q_{r}}\right|_{e_{c}} ^{\tan \Lambda} \\
-\left.N_{\theta}^{T} \frac{\partial m}{\partial y \partial \alpha} \frac{\partial \alpha_{c}}{\partial q_{r}}\right|_{e_{c}}
\end{array}\right] d y \\
& \bar{H}_{q_{r_{j}}}=\int_{0}^{l}\left[\begin{array}{c}
N_{w}^{c} \frac{\pi c}{8} \frac{\partial m}{\partial y} \frac{\partial \alpha}{\partial q_{r} \bar{y}} \tan \Lambda \\
0 \\
-N_{u}^{T} \frac{\pi c}{8} \frac{\partial D}{\partial y} \frac{\partial \alpha}{\partial q_{r} \partial \bar{y}} \tan \Lambda \\
N_{\theta}^{T} \frac{\pi c}{8} \frac{\partial m}{\partial y} \frac{\partial \alpha_{c}}{\partial q_{r} \partial \bar{y}}
\end{array}\right] d y
\end{aligned}
$$




$$
\begin{gathered}
\bar{G}_{q_{r_{j}}}=\int_{0}^{l}\left[\begin{array}{c}
N_{w}^{`} T \frac{\pi e_{m}}{2 V_{\infty}} \frac{\partial m}{\partial y} \frac{\alpha_{c}}{\partial q_{r}} \tan \Lambda_{j} \\
0 \\
-N_{u}^{T} \frac{\pi e_{m}}{2 V_{\infty}} \frac{\partial D}{\partial y} \frac{\alpha_{c}}{\partial q_{r}} \Lambda_{j} \\
N_{\theta}^{T} \frac{\pi e_{m}}{2 V_{\infty}} \frac{\partial m}{\partial y} \frac{\alpha_{c}}{\partial q_{r}} \tan
\end{array}\right] d y \\
\bar{G}_{\alpha_{j}}=\int_{0}^{l}\left[\begin{array}{c}
N_{w}^{T} \frac{\pi e_{m}}{2 V_{\infty}} \frac{\partial m}{\partial y}\left(\frac{c^{2}}{32} \frac{\partial \alpha_{c}}{\partial \alpha \partial \bar{y}}+\left.e_{m} \frac{\partial \alpha_{c}}{\partial \alpha}\right|_{e_{m}}\right) \tan \Lambda_{j} \\
0 \\
-N_{u}^{T} \frac{\pi e_{m}}{2 V_{\infty}} \frac{\partial D}{\partial y}\left(\frac{c^{2}}{32} \frac{\partial \alpha_{c}}{\partial \alpha \partial \bar{y}}+\left.e_{m} \frac{\partial \alpha_{c}}{\partial \alpha}\right|_{e_{m}}\right) \tan \Lambda_{j} \\
N_{\theta}^{T} \frac{\pi e_{m}}{2 V_{\infty}} \frac{\partial m}{\partial y}\left(\frac{c^{2}}{32} \frac{\partial \alpha_{c}}{\partial \alpha \partial \bar{y}}+\left.e_{m} \frac{\partial \alpha_{c}}{\partial \alpha}\right|_{e_{m}}\right) \tan
\end{array}\right] d y
\end{gathered}
$$

where $e_{m}$ is the distance from the quarter chord line the the elastic axis. The component matrices can then be combined together to form the matrices $\bar{G}_{\alpha}, \bar{G}_{q_{r}}, H_{a l p h a}, H_{q_{r}}$, and $\bar{H}_{q_{r}}$ which can be converted to generalized coordinates by multiplying by $\Phi^{T}$.

\section{B. State Equation}

In order to create the state space model we will start by defining the relevant function states to formulate an state equation.

$$
\begin{aligned}
& u_{\alpha}=\left[\alpha, q_{r}\right]^{T} \\
& x_{\Delta}=[\delta, v, w]^{T} \\
& x_{Q}=[q, y, z]^{T}
\end{aligned}
$$

which results in

$$
M_{\ddot{Q} \dot{u}_{\alpha}} \dot{u}_{\alpha}+M_{\ddot{Q} \dot{\Delta} \dot{x_{\Delta}}}+M_{Q \ddot{\Delta}} \ddot{x}_{\Delta}+M_{\ddot{Q} \dot{Q} \dot{x^{\prime}}} \overrightarrow{1}^{0} M_{\ddot{Q} \ddot{Q}} \ddot{X}_{Q}=S_{\ddot{Q} u_{\alpha}} U_{\alpha}+S_{\ddot{Q} \Delta} x_{\Delta}+S_{\ddot{Q} \dot{\Delta}} \dot{x}_{\Delta}+S_{\ddot{Q} Q} x_{Q}+S_{\ddot{Q} \dot{Q}} \dot{x}_{Q}
$$

where,

$$
M_{\ddot{Q}_{\dot{u}_{\alpha}}}=\left[\begin{array}{cc}
-\Phi^{T} \bar{G}_{\alpha} & -\Phi^{T} \bar{G}_{q} \\
0 & 0 \\
0 & 0
\end{array}\right]
$$

where $\bar{G}_{\alpha}$ and $\bar{G}_{q_{r}}$ were determined from Section IV.A.

$$
M_{\ddot{Q} x_{\Delta}}=\left[\begin{array}{ccc}
-\Phi^{T} F_{e c} & 0 & 0 \\
0 & 0 & 0 \\
0 & 0 & 0
\end{array}\right]
$$

where $F_{e c}$ is the circulatory forces from the flaps and is defined in [9].

$$
M_{\ddot{Q} \ddot{Q}}=\left[\begin{array}{ccc}
M_{m S}+\Phi^{T} M_{a n} & 0 & 0 \\
0 & I & 0 \\
0 & 0 & I
\end{array}\right]
$$


where $M_{s}$ is the structural mass matrix whose determination will be discussed earlier in Section III.A and $M_{a n}$ is the non-circulatory aeroelastic contributions of the modal accelerations defined in [9].

$$
S_{\ddot{Q} u_{\alpha}}=\left[\begin{array}{cc}
\Phi^{T} H_{\alpha} & \Phi^{T} H_{q_{r}}+\Phi^{T} \bar{H}_{q_{r}} \\
0 & 0 \\
0 & 0
\end{array}\right]
$$

where $H_{\alpha}$ and $H_{q}$ are the circulatory loads contributed and $\bar{H}_{q}$ is the non-circulatory loads contributed.

$$
S_{\ddot{Q} \Delta}=\left[\begin{array}{ccc}
\Phi^{T}\left(D_{e n}+0.5 D_{e c}+a_{4} \frac{2 V_{\infty}}{c} E_{e c}\right) & \Phi^{T} D_{e c} & \Phi^{T} \frac{2 V_{\infty}}{c} E_{e c} \\
0 & 0 & 0 \\
0 & 0 & 0
\end{array}\right]
$$

where, $D_{e n}$ is the non-circulatory contributes of the flap deflections, $D_{e c}$ is the circulatory contributions, $E_{e c}$ all from [9]. $a_{4}$ is an RT Jones approximation parameter from Table 6 [9].

$$
S_{\ddot{Q} \dot{\Delta}}=\left[\begin{array}{ccc}
\Phi^{T}\left(E_{e n}+0.5 E_{e c}\right) & 0 & 0 \\
0 & 0 & 0 \\
0 & 0 & 0
\end{array}\right]
$$

where $E_{e n}$ is the non-circulatory of the flap deflection rate defined in [9].

$$
S_{\ddot{Q Q}}=\left[\begin{array}{ccc}
-\left(K_{m S}+\Phi^{T}\left(K_{a n}+0.5 K_{a c}+a_{4} \frac{2 V_{\infty}}{c} C_{a c}\right)\right) & -\Phi^{T} K_{a c} & -\Phi^{T} \frac{2 V_{\infty}}{c} C_{a c} \\
0.5 a_{2} \frac{2 V_{\infty} 2}{c}{ }^{2} & -a_{2} \frac{2 V_{\infty}{ }^{2}}{c} & 0 \\
a_{6} \frac{2 \frac{2 \infty}{c}_{c}}{c} & 0 & -\frac{2 V_{\infty}{ }^{2}}{c}
\end{array}\right]
$$

where, $K_{m S}$ is the structural stiffness matrix that was discussed in Section III.A $K_{a n}$ is the non-circulatory load contributions, $K_{a c}$ is the non-circulatory load contributions, $C_{a c}$ is the circulatory load contributions, $a_{2}$ and $a_{6}$ are RT Jones approximation parameters from Table 6 all from in [9].

$$
S_{\ddot{Q} \dot{Q}}=\left[\begin{array}{ccc}
-\left(C_{m S}+\Phi^{T}\left(C_{a n}+0.5 C_{a c}\right)\right) & 0 & 0 \\
a_{4} \frac{2 V_{\infty}}{c} & -a_{3} \frac{2 V_{\infty}}{c} & 0 \\
a_{5} \frac{2 V_{\infty}}{c} & 0 & -a_{3} \frac{2 V_{\infty}}{c}
\end{array}\right]
$$

where, $C_{s}$ is the structural damping matrix using $\zeta=0.01$ defined in Equation $45, C_{a n}$ is the non-circulatory load contributions defined in Equation 128, $a_{3}$ and $a_{5}$ are RT Jones approximation parameters from Table 6 all from in [9].

For the final ASE the input and state vectors will be

$$
U=\left[\begin{array}{llllll}
\alpha & \dot{\alpha} & \ddot{\alpha} & \delta & \dot{\delta} & \ddot{\delta}
\end{array}\right]^{T}
$$

this means that the $\delta$ aeroelastic lag states $v$ and $w$ must be included in the state vector from there Equation $33 \mathrm{can}$ be augment to include the flap aeroelastic lag states.

$$
\begin{aligned}
& x=\left[\begin{array}{ll}
x_{Q} & x_{Q}
\end{array}\right]^{T} \\
& \delta_{\text {lag }}=\left[\begin{array}{ll}
v & w
\end{array}\right]^{T} \\
& \alpha_{g}=\left[\begin{array}{ll}
v & \dot{v}
\end{array}\right]^{T}
\end{aligned}
$$

Equation 33 can be rearranged into 


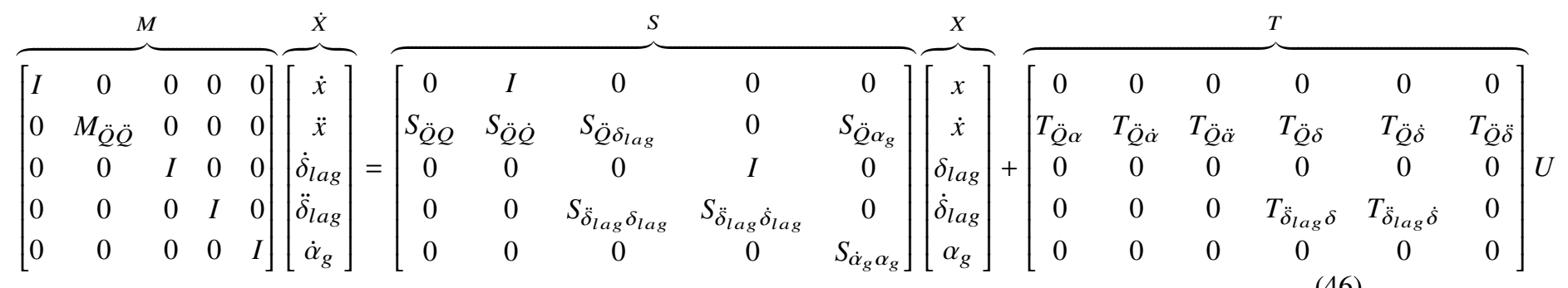

where,

$$
\begin{aligned}
& T_{\ddot{\delta}_{l a g} \delta}=\left[\begin{array}{c}
0.5 a_{2} \frac{2 V_{\infty}{ }^{2}}{c} I \\
a_{6} \frac{2 V_{\infty}{ }^{2}}{c} I
\end{array}\right] \\
& T_{\ddot{\delta}_{\text {lag }} \dot{\delta}}=\left[\begin{array}{l}
a_{4} \frac{2 V_{\infty}}{c} I \\
a_{5} \frac{2 V_{\infty}}{c} I
\end{array}\right] \\
& S_{\ddot{\delta}_{\text {lag }} \delta_{\text {lag }}}=a_{2}{\frac{2 V_{\infty}}{c}}^{2} I \\
& S_{\ddot{\delta}_{\text {lag }} \dot{\delta}_{\text {lag }}}=a_{3} \frac{2 V_{\infty}}{c} I \\
& S_{\ddot{Q} \delta_{l a g}}=\left[\begin{array}{cc}
0 & 0 \\
0 & 0 \\
0 & 0 \\
\Phi^{T} D_{e c} & \Phi^{T} \frac{2 V_{\infty}}{c} E_{e c} \\
0 & 0 \\
0 & 0
\end{array}\right] \\
& S_{\dot{\alpha}_{g} \alpha_{g}}=\left[\begin{array}{cc}
0 & I \\
-a_{2} \frac{2 V_{\infty}}{c} & -a_{3} \frac{2 V_{\infty}}{c}
\end{array}\right] \\
& S_{\ddot{Q}_{g}}=F_{g}
\end{aligned}
$$

where,

$$
F_{g}=\left[\begin{array}{cccccc}
F_{1,1} & 0 & 0 & \ldots & 0 & 0 \\
F_{2,1} & F_{2,2} & 0 & \ldots & 0 & 0 \\
0 & F_{3,2} & F_{3,3} & \ldots & 0 & 0 \\
0 & 0 & F_{4,3} & & 0 & 0 \\
\vdots & \vdots & \vdots & \ddots & \vdots & \vdots \\
0 & 0 & 0 & \ldots & F_{m-1, m-1} & F_{m-1, m} \\
0 & 0 & 0 & \ldots & 0 & F_{m, m}
\end{array}\right]
$$

where $m$ is the number of nodes in the wing finite element model and

$$
\left[\begin{array}{c}
F_{i, i} \\
F_{i+1, i}
\end{array}\right]=\int_{0}^{l}\left[\begin{array}{c}
N_{w}^{T} l_{\alpha_{g}}-N_{w}^{\prime T} m_{\alpha_{g}} \tan \Lambda \\
N_{v}^{T} d_{\alpha_{g}} \\
N_{u}^{T} d_{\alpha_{g}} \tan \Lambda \\
-N_{\theta}^{T} m_{\alpha_{g}}
\end{array}\right] d x
$$


where the $N_{j}$ 's are the Hermite shape function coefficients and

$$
\begin{gathered}
l_{\alpha_{g}}=c_{L_{\alpha}} Q_{\infty} C \frac{d l}{d y} \frac{1}{\cos \Lambda} \\
d_{\alpha_{g}}=\frac{1}{2 C_{L}} \frac{C_{D_{\alpha}}}{C_{L_{\alpha}}} c_{L_{\alpha}} Q_{\infty} C \frac{d l}{d y} \frac{1}{\cos \Lambda} \\
m_{\alpha_{g}}=\frac{e}{c} c_{L_{\alpha}} Q_{\infty} C \frac{d l}{d y} \frac{1}{\cos \Lambda}
\end{gathered}
$$

when the clamped boundary condition is applied

$$
\begin{aligned}
& F_{g}=F_{g}(7: \text { end }, 7: \text { end }) ; \\
& T_{\ddot{Q} \alpha}=\left[\begin{array}{c}
0 \\
0 \\
0 \\
\Phi^{T} H_{\alpha} \\
0 \\
0
\end{array}\right] \\
& T_{\ddot{Q} \dot{\alpha}}=\left[\begin{array}{c}
0 \\
0 \\
0 \\
\Phi^{T}\left(H_{q_{r}}+\bar{H}_{q_{r}}+\bar{G}_{\alpha}\right) \\
0 \\
0
\end{array}\right] \\
& T_{\ddot{Q} \ddot{\alpha}}=\left[\begin{array}{c}
0 \\
0 \\
0 \\
\Phi^{T} \bar{G}_{q_{r}} \\
0 \\
0
\end{array}\right] \\
& T_{\ddot{Q} \delta}=\left[\begin{array}{c}
0 \\
0 \\
0 \\
\Phi^{T}\left(D_{e n}+0.5 D_{e c}+a_{4} \frac{2 V_{\infty}}{c} E_{e c}\right) \\
0 \\
0
\end{array}\right] \\
& T_{\ddot{Q} \dot{\delta}}=\left[\begin{array}{c}
0 \\
0 \\
0 \\
\Phi^{T} F_{e n} \\
0 \\
0
\end{array}\right]
\end{aligned}
$$




$$
T_{\ddot{Q} \ddot{\delta}}=\left[\begin{array}{c}
0 \\
0 \\
0 \\
\Phi^{T}\left(E_{e n}+0.5 E_{e c}\right) \\
0 \\
0
\end{array}\right]
$$

Equation 46 can be distilled to

$$
M \dot{X}=S X+T U
$$

Then the final ASE can be found by left multiplying the inversion of the cumulative mass matrix $M$ and adding in the gust vector $w_{g}$ as a disturbance.

$$
\dot{X}=\overbrace{M^{-1} S}^{A} X+\overbrace{M^{-1} T}^{B} U+E w_{g}
$$

where

$$
w_{g}=\left[\begin{array}{ll}
g & \dot{g}
\end{array}\right]^{T}
$$

and the gust matrix $E$ is

$$
E=M^{-1}\left[\begin{array}{cc}
0 & 0 \\
0 & 0 \\
0 & 0 \\
0 & 0 \\
a_{2} \frac{2 V_{\infty}}{c} \frac{1}{V_{\infty}} & a_{1} \frac{2 V_{\infty}}{c} \frac{1}{V_{\infty}}
\end{array}\right]
$$

and a final ASE of

$$
\dot{X}=A X+B U+E w_{g}
$$

\section{Output Equation}

The output bears many similarities to the state equation development and focuses on the translation of the model states to measurable quantities. The first set of desired measurements are the element displacements, velocities, and accelerations.

$$
y_{e}=\left[\begin{array}{lllllll}
z_{e}(y) & \phi_{z_{e}}(y) & x_{e} & \phi_{x_{e}}(y) & \theta_{e}(y) & \phi_{\theta_{e}}(y) & z_{e}(y)
\end{array}\right]^{T}
$$

where,

$$
\begin{aligned}
& y_{e}=\Phi C_{1} X \\
& \dot{y}_{e}=\Phi C_{2} X
\end{aligned}
$$

and

$$
\ddot{y}_{e}=\Phi C_{2}\left(A X+B U+E w_{g}\right)
$$

where $C_{1}$ is the selector matrix for $q$ states and $C_{2}$ is the selector matrix for $\dot{q}$.

\section{Hinge Moment Output Equation}

For easy access to the hinge moments the hinge moments due to ASE states have been provided as an output.

$$
y_{h}=\left[\begin{array}{llllll}
M_{h_{1 B}} & \ldots & M_{h_{6 B}} & M_{h_{1 A}} & \ldots & M_{h_{6 A}}
\end{array}\right]^{T}
$$

The output hinge equation is

$$
y_{h}=H_{X} X+H_{U} U
$$

where, 


$$
H_{X}=\left[\begin{array}{ll}
H_{e} & H_{\text {elag }}+H_{\text {lag }}
\end{array}\right]
$$

and,

$$
H_{e}=\left[\begin{array}{llllll}
D_{\delta_{n}}+0.5 D_{\delta_{c}}+a_{4} \frac{2 V_{\infty}}{c} E_{\delta_{c}} & D_{\delta_{c}} & \frac{2 V_{\infty}}{c} E_{\delta_{c}} & E_{\delta_{n}}+0.5 E_{\delta_{c}} & 0 & 0
\end{array}\right]+V_{\delta n} C_{2} A C_{3}
$$

where $V_{\delta n}$ is the non circulatory elemental force contributions from the elastic states to the hinge moment defined in [9], $C_{3}$ is the selector matrix for the elastic states $\left[\begin{array}{ll}x & \dot{x}\end{array}\right]^{T}$

$$
H_{\text {elag }}=V_{\delta n} C_{2} A C_{4}
$$

where $C_{4}$ is the selector matrix for the flap lag states $\left[\begin{array}{ll}\dot{\delta}_{l a g} & \ddot{\delta}_{l a g}\end{array}\right]^{T}$

$$
H_{\text {lag }}=\left[\begin{array}{ll}
-C_{5} K_{\delta} C_{6} & -C_{5} C_{\delta} C_{6}
\end{array}\right]+-C_{5} M_{\delta} C_{6} C_{2} A C_{3}
$$

where, $C_{5}$ is the output selector for the flap lag states and $C_{6}$ is the input selector matrix for the same set of states. $K_{\delta}$ is the flap stiffness matrix, $C_{\delta}$ is the flap damping matrix, $M_{\delta}$ is the flap mass matrix.

$$
H_{U}=\left[\begin{array}{ll}
H_{U_{\alpha}} & H_{U_{\delta}}
\end{array}\right]
$$

and,

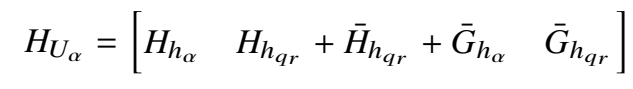

where $H_{h_{\alpha}}$ is the circulatory hinge moment contributions of the angle of attack, $H_{h_{q r}}$ is the circulatory hinge moment contributions of the pitch rate, $\bar{H}_{h_{q r}}$ is the non-circulatory hinge moment contributions of the pitch rate, $\bar{G}_{h_{\alpha}}$ is the non-circulatory mass hinge moment contributions of the pitch rate, $\bar{G}_{h_{q r}}$ is the non-circulatory hinge moment contributions of the angle of attack acceleration.

$$
H_{U_{\delta}}=\left[\begin{array}{lll}
-C_{5} K_{\delta} C_{7} & -C_{5} C_{\delta} C_{7} & F_{\delta n}
\end{array}\right]
$$

where $C_{7}$ is the input flap selection matrix and $F_{\delta n}$ is the aerodynamic forces associated with the circulatory acceleration of the flaps.

\section{Wing Force Output Equation}

Starting from Equation 33 we can define

$$
M_{s} \ddot{q}+C_{s} \dot{q}+K_{s} q=F_{q}
$$

where $F_{q}$ is the force being applied to the wing structure represented by the left side of the equation. Isolating the modal forces results in

$$
F_{q}=\begin{gathered}
-M_{a n} \ddot{q}-\left(K_{a n}+0.5 K_{a c}+a_{4} \frac{2 V_{\infty}}{c}\right) q-K_{a c} y-\frac{2 V_{c}}{c} C_{a c} z-\left(C_{a n}+0.5 C_{a c}\right) \dot{q}+\left[\begin{array}{ll}
H_{\alpha} & H_{q_{r}}+\bar{H}_{q_{r}}
\end{array}\right] u_{\alpha}+ \\
\left(D_{e n}+0.5 D_{e c}+a_{4} \frac{2 V_{\infty}}{c} E_{e c}\right) \delta+D_{e c} v+\frac{2 V_{\infty}}{c} E_{e c} w+F_{e n} \ddot{\delta}+\left[\begin{array}{ll}
\bar{G}_{\alpha} & \bar{G}_{q}
\end{array}\right] \dot{u}_{\alpha}+\left(E_{e n}+0.5 E_{e c}\right) \dot{\delta}
\end{gathered}
$$

The modal forces can then be translated to general coordinates via

$$
F=\Phi F_{q}
$$

The root reaction forces and moments then become

$$
\begin{aligned}
& R_{x}=-\sum_{y_{\text {root }}}^{y_{\text {tip }}} F_{x}(y) \\
& R_{z}=-\sum_{y_{\text {root }}}^{y_{\text {tip }}} F_{z}(y)
\end{aligned}
$$




$$
\begin{aligned}
& M_{x}=-\sum_{y_{\text {root }}}^{y_{\text {tip }}} M_{z}(y)+F_{z}(y) y \\
& M_{z}=-\sum_{y_{\text {root }}}^{y_{\text {tip }}} M_{x}(y)+F_{x}(y) y
\end{aligned}
$$

These forces and moments need to be translated back to the ASE state and input state vectors

$$
F=F_{x}+F_{u}+F_{w}
$$

where,

$$
F_{m a}=M_{a} \Phi C_{2} A
$$

where,

$$
M_{a n}=\Phi^{T} M_{a} \Phi
$$

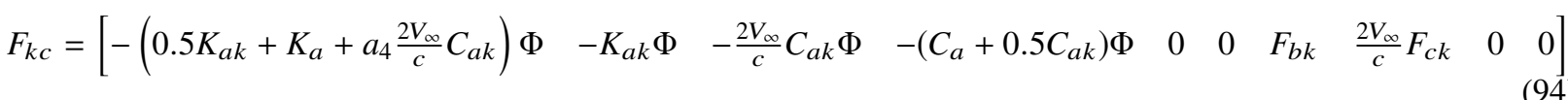
where,

$$
\begin{gathered}
K_{a n}=\Phi^{T} K_{a} \Phi \\
K_{a c}=\Phi^{T} K_{a k} \Phi \\
C_{a c}=\Phi^{T} C_{a k} \Phi \\
D_{e c}=\Phi^{T} F_{b k} \\
E_{e c}=\Phi^{T} F_{c k}
\end{gathered}
$$

Then combining Equations 92 and 94 result in

$$
F_{x}=F_{m a}+F_{k c}
$$

The real coordinate forces from the angle of attack inputs $u_{\alpha}=\left[\begin{array}{ccc}\alpha & \dot{\alpha} & \ddot{\alpha}\end{array}\right]$ are

$$
F_{u_{\alpha}}=\left[\begin{array}{lll}
H_{\theta} & H_{\dot{\theta}}+\bar{H}_{\dot{\theta}}+G_{\theta} & G_{\dot{\theta}}
\end{array}\right]
$$

where,

$$
\begin{aligned}
H_{\alpha} & =\Phi^{T} H_{\theta} \\
H_{q_{r}} & =\Phi^{T} H_{\dot{\theta}} \\
\bar{H}_{q_{r}} & =\Phi^{T} H_{\dot{\theta}} \\
G_{\alpha} & =\Phi^{T} G_{\theta} \\
G_{q_{r}} & =\Phi^{T} G_{\dot{\theta}}
\end{aligned}
$$

The real coordinate forces from the flap inputs are

$$
F_{\delta}=F_{b}+0.5 F_{b k}+a_{4} \frac{2 V_{\infty}}{c} F_{c k}
$$

where,

$$
\begin{aligned}
& D_{e n}=\Phi F_{b} \\
& D_{e c}=\Phi F_{b k} \\
& E_{e c}=\Phi F_{c k}
\end{aligned}
$$

The real coordinate forces form the flap rates are

$$
F_{\dot{\delta}}=F_{c}+0.5 F_{c k}
$$




$$
\begin{gathered}
E_{e n}=\Phi F_{c} \\
E_{e c}=\Phi F_{c k}
\end{gathered}
$$

The real coordinates from the flap accelerations

$$
F_{\ddot{\delta}}=F_{d}
$$

where,

$$
F_{\text {en }}=\Phi F_{d}
$$

These combine to create

$$
F_{u}=\left[\begin{array}{llll}
F_{u \alpha} & F_{\delta} & F_{\dot{\delta}} & F_{\ddot{\delta}}
\end{array}\right]
$$

The force contributions from gust are

$$
F_{w}=\frac{1}{V_{\infty}}\left[\begin{array}{ll}
H_{\theta} & H_{\dot{\theta}}+G_{\theta}
\end{array}\right]
$$

\section{Strain Gauge Output}

The translation of the moments from the the previous section to strain gauge outputs is inversely proportional to $\frac{1}{E I}$ resulting in the output equation

$$
\epsilon=S_{G} M_{x} X+S_{G} M_{u} U+S_{G} M_{w} w_{g}
$$

where,

$$
S_{G}=\left[\begin{array}{ccccc}
\frac{-1}{E_{1} I_{1}} & 0 & \ldots & 0 & \\
0 & \frac{-1}{E_{2} I_{2}} & \ldots & 0 & \\
\vdots & \vdots & \ddots & \vdots & \vdots \\
0 & 0 & \cdots & \frac{-1}{E_{m} I_{m}} &
\end{array}\right]
$$

\section{Offset IMUs}

Any desired IMU that is offset from the elastic axis can be created via the current $C, D$, and $F$ matrices and the general geometry of their locations, for now we will assume that the IMUs have no $z$ displacement from the neutral axis. We are also going to assume that gravity has already been removed from the accelerometer readings. Starting with the knowledge that

$$
\Delta z=z-z_{\text {trim }}
$$

and

$$
\Delta x=x-x_{\text {trim }}
$$

where $\Delta z$ and $\Delta x$ are the states we are interested and the $x_{\text {trim }}$ and $z_{\text {trim }}$ are the deflected trim states that the ASE is centered about and are effectively zeroed out by the model construction. From here knowing that we are placing the IMU's on the z-plane of the elastic axis the arbitrary IMU positions would be

$$
\begin{gathered}
\Delta z=\Delta z_{o}+d \sin \left(\theta_{o}\right) \\
\Delta x=\Delta x_{o}+d\left(\cos \left(\theta_{o}\right)-1\right)
\end{gathered}
$$

where the parameters with sub $o$ are the origin points at the elastic axis and $d$ is the horizontal x distance from the elastic axis, positive in the forward direction and negative in the aft direction. This results in nonlinear acceleration values of

$$
\ddot{\Delta z}=\ddot{z}_{o}+d \ddot{\theta}_{o} \cos \left(\theta_{o}\right)-d \dot{\theta}_{o}^{2} \sin \left(\theta_{o}\right)
$$

and

$$
\ddot{\Delta x}=\ddot{x}_{o}-d \ddot{\theta}_{o} \sin \left(\theta_{o}\right)-d \dot{\theta}_{o}^{2} \cos \left(\theta_{o}\right)
$$

which are linearized to 


$$
\Delta z=\ddot{z}_{o}+d \ddot{\theta}_{o}
$$

and

$$
\Delta \ddot{x}=\ddot{x}_{o}
$$

which can be used to create an offset IMU matrix

$$
C_{I M U}=\left[\begin{array}{ccccc}
\cdots & \Delta z_{z_{o}}\left(y_{p o s}\right) & \cdots & \Delta z_{\theta_{o}}\left(y_{p o s}\right) & \cdots \\
\cdots & \Delta x_{x_{o}}\left(y_{p o s}\right) & \cdots & \cdots & \cdots
\end{array}\right]
$$

and can augment the output $y$

$$
\left[\begin{array}{c}
y \\
y_{\text {aug }}
\end{array}\right]=\left[\begin{array}{c}
C \\
C_{I M U} C
\end{array}\right] X+\left[\begin{array}{c}
D \\
C_{I M U} D
\end{array}\right] U+\left[\begin{array}{c}
F \\
C_{I M U} F
\end{array}\right] d
$$

\section{Simulation Results}

The initial simulations are using an simple sinusoidal gust input at one hertz with a peak magnitude of $2 \mathrm{fps}$. The wind speed is $91.5 f p s$ at an dynamic pressure of $10 p s f$. A simple full state LQR controller was used to demonstrate the models implementation and ability for simple controller implementation. The weightings were uniformly applied where $Q=1^{-6} I$ and $R=1^{-5}$. The simulation was constructed in Simulink with a fourth order Runge-Kutta integration method with a time step of $5 \mathrm{~ms}$.

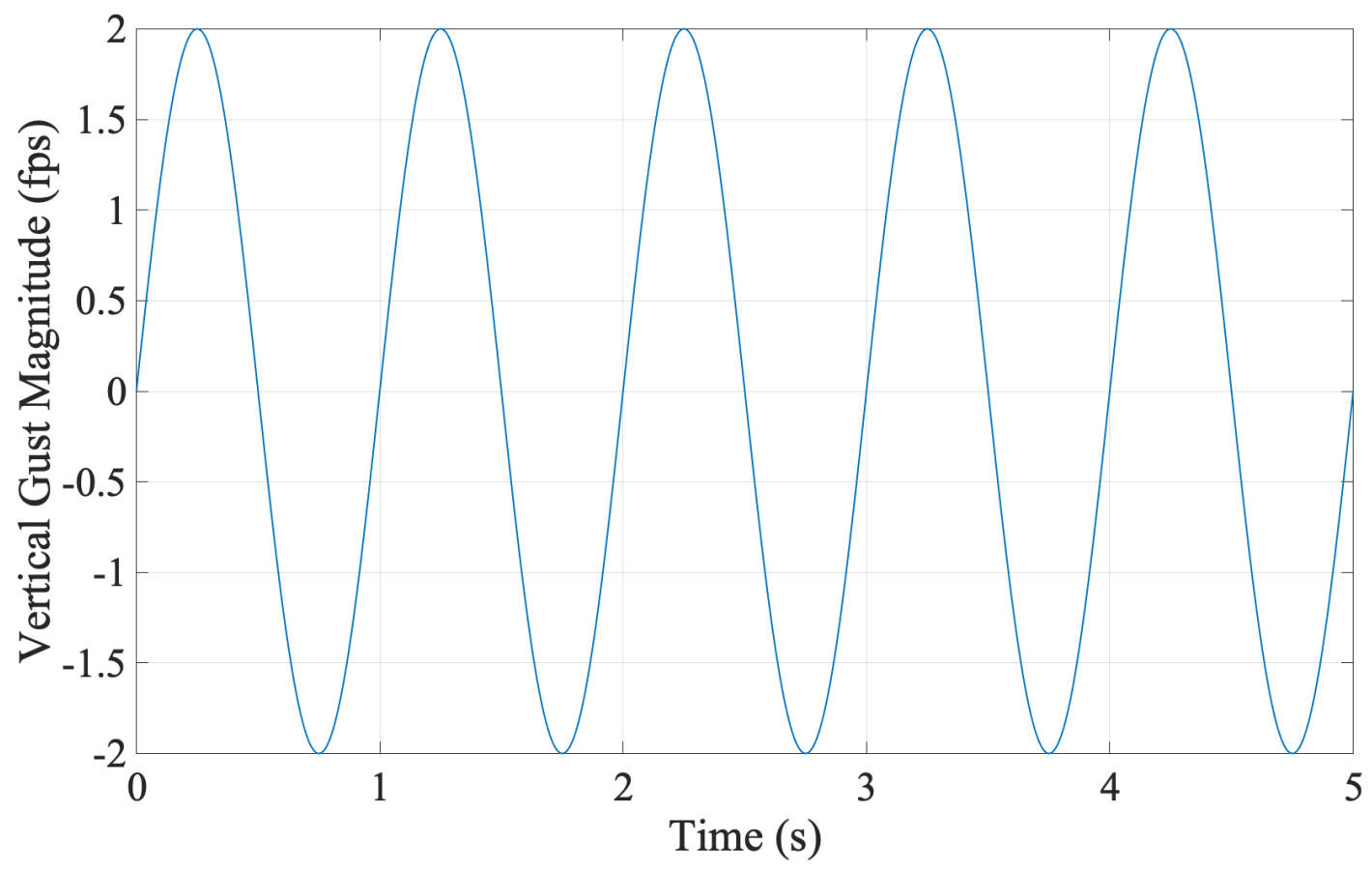

Fig. 4 Input sinusoidal gust

Figure 4 shows the input gust and Figure 5 shows how that gust input gets distributed to the individual gust channels and includes a time delay for the wing sweep. 


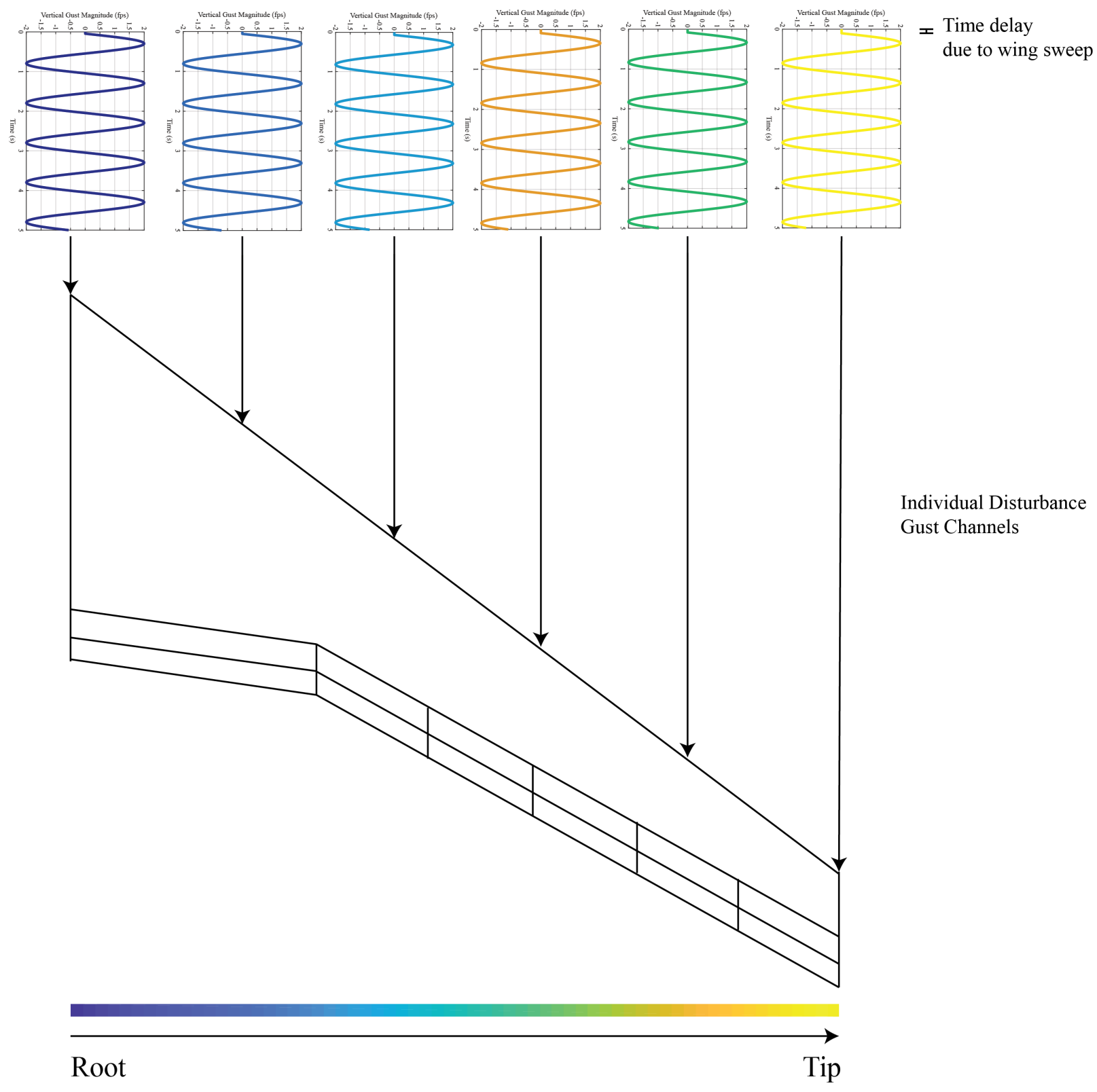

Fig. 5 Gust propagation model from the initial gust in Figure 4 with the time delay due to the wing sweep and demonstrating the local gust channels

Figure 6 shows the wing tip displacement with and with out the LQR control demonstrating a reduction of the effect the gust has on the tip displacement. 


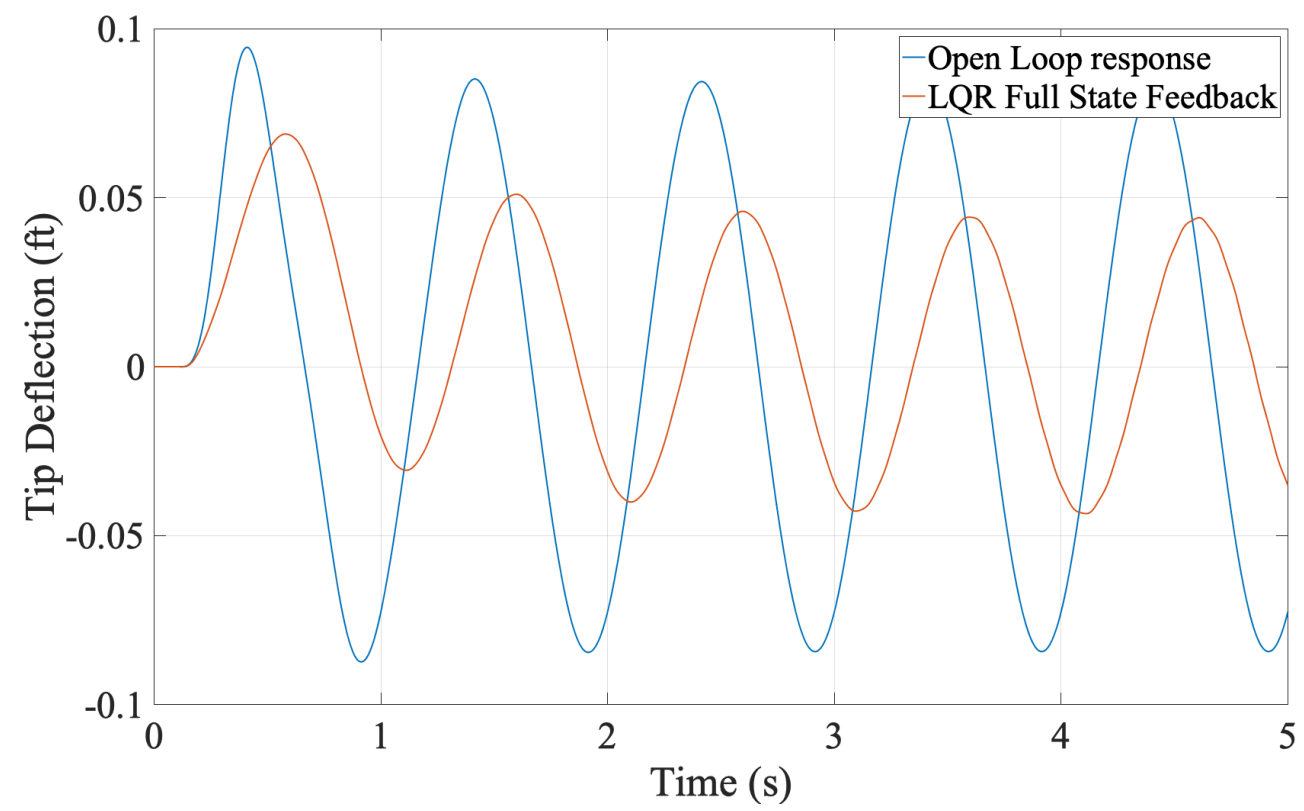

Fig. 6 Model wing tip displacement open loop and full state feedback gust response

Figure 7 shows the root bending with and with out the LQR control demonstrating a interesting phenomena of the uniform weightings where initially the LQR controller increased the root bending moment but eventually decreased it over all. In a more realistic implementation the root bending moment would likely need much higher weighting.

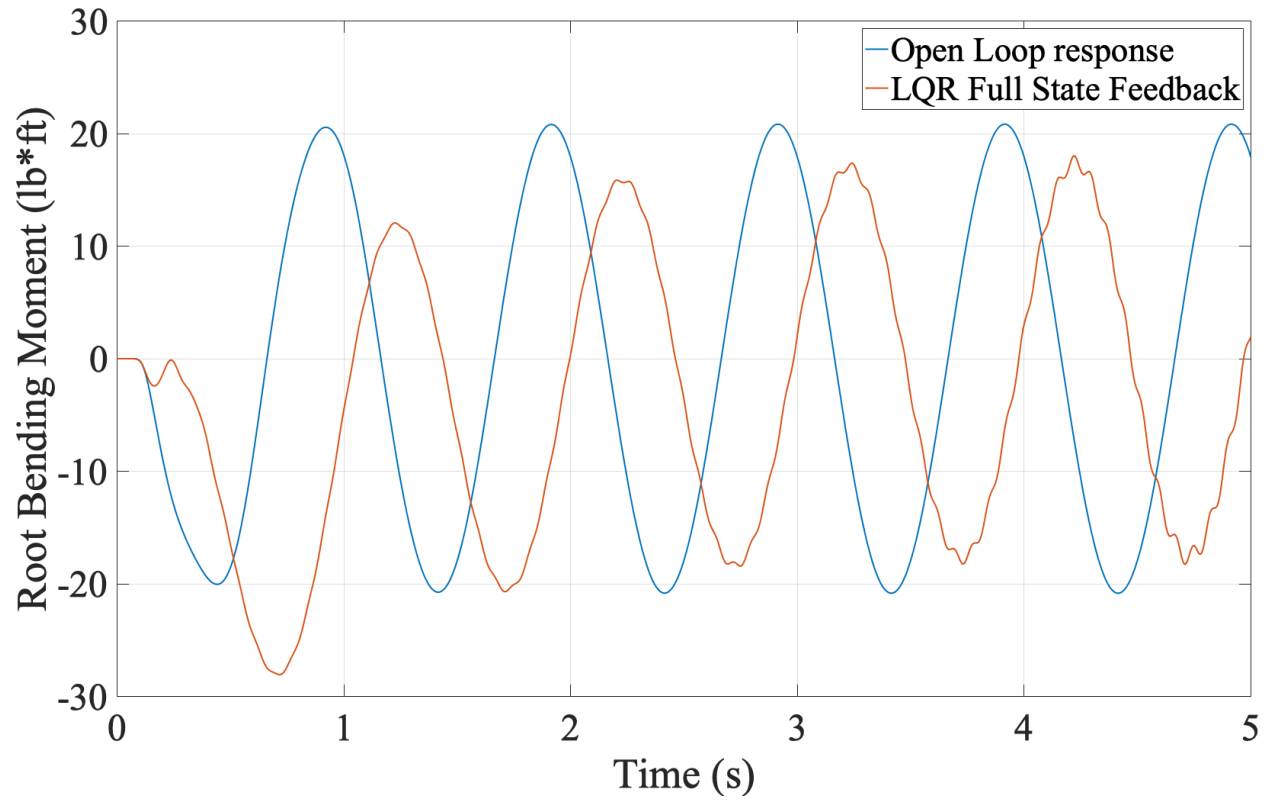

Fig. 7 Root bending moment open loop and full state feedback gust response

Figure 8 shows the control response for each of the individual flaps in the simulation. This shows the location of each flap and the responses are fairly similar with the B flaps having smaller responses because their smaller control surface area and the uniform weighting applied to the LQR controller. 

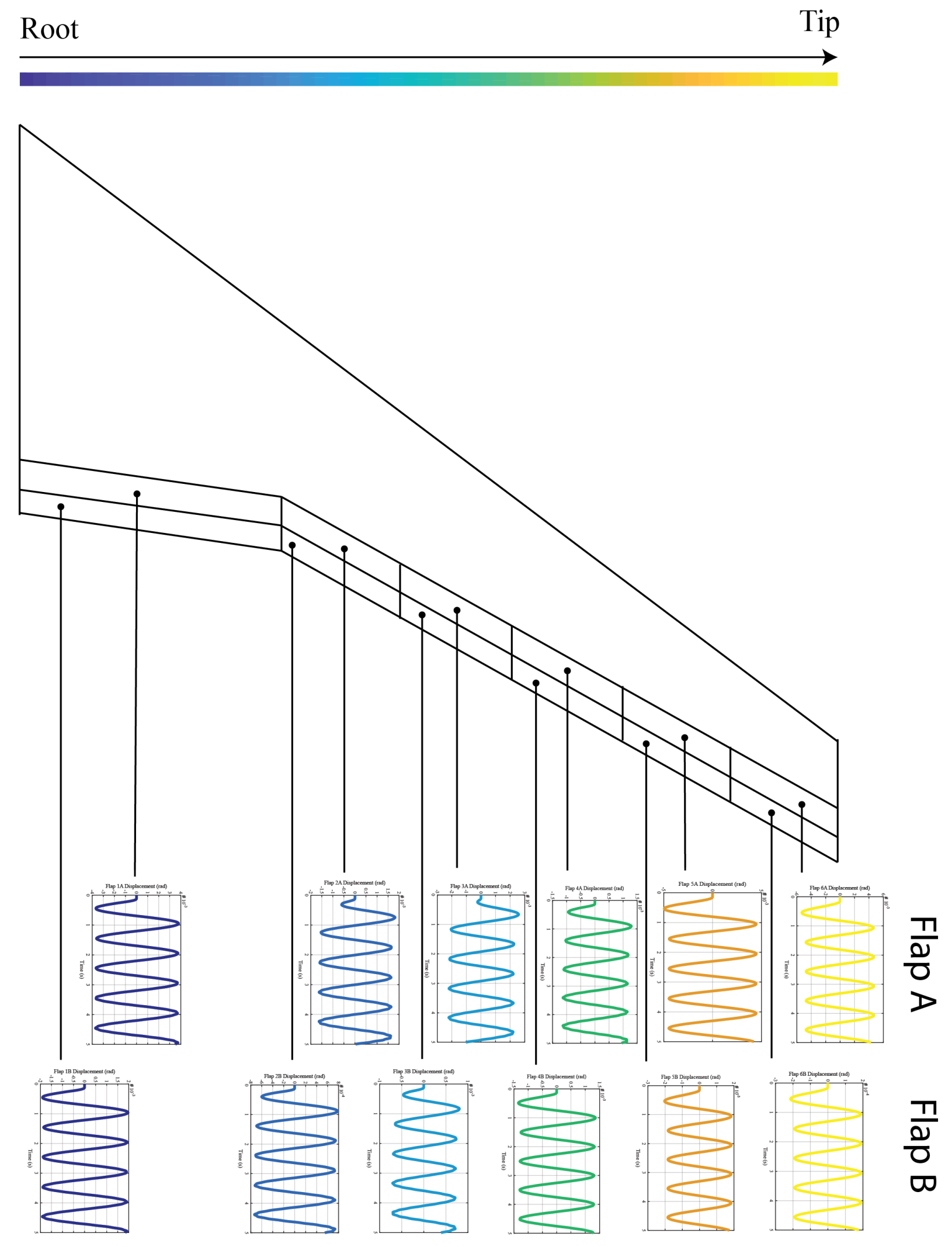

Fig. 8 Individual flap response for the LQR controller 


\section{Conclusion}

We have developed an aeroservoelastic model with gust inputs that vary accross the wing span.We then demonstrated its ability to perform simple control simulations. This showed the accessibility of the various experimental outputs and easy of control integration into the mode. The model was compared to previous physical models with good structural agreement on the lower order modes.

\section{Acknowledgements}

The authors wish to acknowledge NASA Advanced Air Transport Technologies project for the funding support of this work.

\section{References}

[1] Hoblit, F. M., Gust loads on aircraft: concepts and applications, American Institute of Aeronautics and Astronautics, 1988.

[2] Regan, C. D., and Jutte, C. V., "Survey of applications of active control technology for gust alleviation and new challenges for lighter-weight aircraft," 2012.

[3] Giesseler, H.-G., Kopf, M., Varutti, P., Faulwasser, T., and Findeisen, R., "Model predictive control for gust load alleviation," IFAC Proceedings Volumes, Vol. 45, No. 17, 2012, pp. 27-32.

[4] Giesseler, H.-G., Kopf, M., Faulwasser, T., Varutti, P., and Findeisen, R., "Gust load alleviation based on model predictive control," Tech. rep., 2013.

[5] Nguyen, N. T., Livne, E., Precup, N., Urnes, J. M., Nelson, C., Ting, E., and Lebofsky, S., "Experimental investigation of a flexible wing with a variable camber continuous trailing edge flap design," 32nd AIAA Applied Aerodynamics Conference, 2014, p. 2441.

[6] Drew, M. C., Hashemi, K. E., Cramer, N. B., and Nguyen, N. T., "Multi-objective Optimal Control of the 6-DoF Aeroservoelastic Common Research Model with Aspect Ratio 13.5 Wing,” AIAA Scitech 2019 Forum, 2019, p. 0220.

[7] Rivers, M. B., and Dittberner, A., "Experimental investigations of the NASA common research model," Journal of Aircraft, Vol. 51, No. 4, 2014, pp. 1183-1193.

[8] Precup, N., Mor, M., and Livne, E., "An Active Variable Camber Continuous Trailing Edge Flapped Wing Wind Tunnel Model for Aeroelastic" In-Flight" Shape Optimization Tests," 2018 Multidisciplinary Analysis and Optimization Conference, $2018, \mathrm{p}$. 3106.

[9] Ting, E., Chaparro, D., and Nguyen, N. T., "Development of an Integrated Nonlinear Aeroservoelastic Flight Dynamic Model of the Truss-Braced Wing Aircraft," 58th AIAA/ASCE/AHS/ASC Structures, Structural Dynamics, and Materials Conference, 2017, p. 1815.

[10] Nguyen, N. T., Ting, E., and Chaparro, D., "Development of an Integrated Nonlinear Aeroservoelastic Flight Dynamic Model of the NASA Generic Transport Model," 2018 AIAA/ASCE/AHS/ASC Structures, Structural Dynamics, and Materials Conference, 2018, p. 2210.

[11] Nguyen, N. T., Ting, E., and Lebofsky, S., "Inertial Force Coupling to Nonlinear Aeroelasticity of Flexible Wing Aircraft," 15th Dynamics Specialists Conference, 2016, p. 1094. 